\title{
A Modified Patients' Posture for PICC Catheter Placement Makes Patients more Comfortable and Relaxed
}

T Li, YZ Yan, D Wei, F Bilong

\begin{abstract}
Objective: This paper aimed to evaluate a modified posture for PICC (peripherally inserted central catheter) catheter placement
\end{abstract} by comparing with traditional posture in terms of procedure-related complications and catheter heterotopia.

Method: From December 2006 to December 2008, 519 patients were recruited into control group. These patients received PICC insertion by holding the traditional posture, i.e., horizontal position, arm abduced about $90^{\circ}$, and when the needle successfully entered vein, patients were required to turn his/her head to the procedure side and touch his/her chin to clavicle until the catheter reached the expected position. From January 2009 to June 2010, 508 patients to receive PICC insertion were recruited to the experiment group by holding a modified posture, in which the patients hold horizontal position, arm abduces about $90^{\circ}$, with no other specific requirement during the insertion procedure.

Results: No significant differences were found between the traditional and the modified posture group in the incidence of catheter heterotopia and other procedure-related complications.

Conclusion Modified posture makes patients more comfortable and relaxed during the PICC procedure. And it's especially more applicable for non-cooperative patients.

Keywords: Catheter heterotopia, modified posture, PICC

From: Comprehensive Medical Department of Wuhan ZhongNan Hospital of Wuchan University, Wuhan 430071, PR China.

Correspondence: $\quad$ Dr T Li, 169 East Lake Road, Wuchang District, Wuhan City, Hubei Province, 430071, China. Fax: +8610 027.67812999, e-mail: tongli0614@ sina.com 


\section{INTRODUCTION}

PICC (peripherally inserted central catheter) is an intravenous procedure to reach superior vena cava through peripheral veins, including median cubital vein, basilic vein and cephalic vein(1).

PICCs can be used for intravenous delivery of chemotherapy regimens, antibiotics or other medications Si.nce it can transfuse stimulant medicine safely and ease pain, PICC provides a relative painless transfusion passage for patients(2).

Studies revealed that patients with PICC had high satisfication(3,4), and application of PICC presented strong clinical value ${ }^{5}$. However, PICC catheter placement has its limitations, especially for those non-cooperative patients, and catheter heterotopia could also happen and it needs operators to pay special attention to.

\section{Background}

PICC is widely used as an intravenous access to superior vena cava, and it's usually performed by certified registered nurses due to its relative high risk. When catheter tip is not located in superior vena cava it is called catheter heterotopia, in which the tip is usually located in jugular vein, axillary vein or right atrium6. The incidence of catheter heterotopia is about $12.5 \%$ (7), and it's usually related with vascular disease, improper body posture, insertion pathway (cephalic 
vein) and mediastinal mass 8. Catheter heterotopia contributes to complications of PICC (9) and can even lead to paravertebral hydrops and atrial fibrillation (10).

In traditional PICC operation, when the needle successfully entered vein, patients are required to turn his/her head to the procedure side and move his/her chin to touch clavicle to interdict jugular vein until the catheter gets to the expected position (11-15) in order to avoid catheter heterotopias. However, patients with dementia, Parkinson disease, cerebral infarction and mental disorder are difficult to cooperate with operators to finish this procedure. Even with cooperative patients, it's also very difficult to make the patients feel comfortable with this posture.

This study compared a modified patient posture for PICC catheter placement with a traditional posture which had been widely used in China, and checked the incidence of catheter heterotopia and followed up with the complications caused by catheter insertion. The results suggested that the modified patient posture for PICC catheter placement could make patients more comfortable and relaxed during the procedure without increasing rates of complications and is worth being utilized more in the clinical practice. Meanwhile, we analyzed potential reasons for catheter heterotopia and suggested some precautions before catheter insertion. 


\title{
The study
}

\begin{abstract}
Aim
The aim of the study was to evaluate a modified posture for PICC catheter placement by comparing with traditional posture in complications and catheter heterotopia in a Chinese population.
\end{abstract}

\section{Design}

The participants to receive PICC catheter placement were recruited from December 2006 to June 2010 and their clinical information and follow-up data were collected for analysis.

\section{Participants}

From December 2006 to June 2010, 1027 patients to receive PICC catheter placement were recruited into study. They were from general internal medicine department, oncology department, neurosurgery department, general surgery department and hematology department in ZhongNan hospital of Wuhan University. All patients enrolled into study of PICC catheter placement signed informed consent. In the control group, 519 patients holding traditional posture for catheter insertion were recruited from December 2006 to December 2008. In experiment group, 508 
patients holding modified posture for catheter insertion were recruited from January 2009 to June 2010.

\section{Procedure}

The puncture kits were from BD company (4F or 5F, BD First PICC, BD infusion therapy system Inc, USA). All the placement procedures were performed by certified registered nurses. The patients in the control group took traditional PICC posture, i.e. horizontal position, arm abduces about $90^{\circ}$, and when the when the needle successfully entered vein, patients turned his/her head to procedure side and moved chin to touch clavicle until the catheter reached the expected position. The patients in experiment group took modified posture, i.e. horizontal position, arm abduces about $90^{\circ}$, for those patients who can't fulfill this action can put their arm freely, and when the cathether was being inserted, patients can hold their comfortable and relaxed posture without the requirements mentioned for patients in control group.

\section{Data collection}

Data of the 1027 participants from different departments were collected, starting from enrollment into the in-patient departments of the hospital. Variables collected included gender, age, 
diagnosis, concomitant diseases, key information related to PICC catheter placement (inserted arm, inserted vein, and treatment protocol), incidence and position of catheter heterotopia by $\mathrm{X}$-ray location after catheter insertion, and incidence of complications and catheter retention time during follow-up visit. The participants were also asked about their feeling during the catheter insertion.

\section{Ethical considerations}

The study was approved by the appropriate ethics committee. Participants were informed that participation was voluntary and they could withdraw at any time. They signed consent forms after having received written and oral information about the study and none withdrew from the study.

\section{Data analysis}

The data analysis technicians are blind to the data sources. All data were analyzed using SPSS

13.0 (SPSS Inc., Chicago, IL, USA) statistical package. Chi-square tests were used to determine 
whether there is a significant difference between the expected frequencies and the observed frequencies in the two groups. Statistical significance level was set at $p<0.05$.

\section{RESULTS}

\section{Patient distribution and general information of participants from control and experiment}

\section{group}

The study participants were recruited from general internal medicine department, oncology department, neurosurgery department, general surgery department and hematology department in ZhongNan Hospital of Wuhan University. There was no statistical significant difference with regard to the distribution of departments between participants in control and experiment group (shown in Table 1). And follow-up data showed that the catheter retention time was quite similar in two groups. There are 519 patients in the control group and catheter retention time ranged from 7 to 366 days, with mean time being 305 days, while there were 508 patients in the experiment group and catheter retention time ranged from 10 days to 456 days, with mean time being 360 days. 
There was also no significant difference in participants' age distribution between the two groups,

$51.38 \%$ versus $49.92 \%$ were less than 50 years old, and $48.62 \%$ versus $50.08 \%$ were 50 or older in control and experimental group respectively. Furthermore, gender distribution, catheter insertion arm, insertion vein and treatment protocols were also comparable between control group and experiment group. Detailed information was shown in table 1 and 2.

\section{Complications and incidence of catheter heterotopia of patients between control and experiment group}

Complications and incidence \& positions of catheter heterotopia caused by PICC catheter placement was recorded in our study. The complications were phlebitis, catheter obstruction, skin allergy, catheter emersion, infections and insertion problems. The complication incidence in modified posture group was even lower in experiment group (4.92\%) than that in control group (7.71\%). However the statistical analysis showed that the difference is not statistically significant $(p=0.800)$.

Catheter heterotopia was another main problem in PICC catheter placement. In our study, the incidence of catheter heterotopias was lower than mean level $12.5 \%$ reported by other study(4).Confirmed by X-ray radiograph, positions of heterotopia were jungular vein, axillary vein, right atrium and twist. There was no statistical difference in complication types between 
two groups. More information were showed in table 3

\section{DISCUSSION}

This study recruited 1027 participants from various inpatient departments in ZhongNan Hospital of Wuhan University to evaluate a modified patient posture for PICC catheter placement. In the control group, 519 patients receiving traditional posture for catheter placement were recruited from December 2006 to December 2008. In the experiment group, 508 patients receiving modified posture for catheter placement were recruited from January 2009 to June 2010. The participants in the two groups were generally comparable, and they showed similar incidence of complications and catheter heterotopias. Admittedly, a better design would be data on the two groups at the same time frame.

\section{Effect of body posture on incidence of catheter heterotopia and complications}

During the insertion operation, when catheters are placed in shoulder, patients in control group are required to turn his/her head to the procedure side and move his/her chin to touch clavicle to avoid catheter heterotopia through jugular vein. However, patients with dementia, Parkinson 
disease, cerebral infarction and other mental disorders usually couldn't keep the special posture to cooperate with the operators to finish this procedure. Some patients even felt anxiety after they

posed that way. And anxiety could stimulate sympathetic nerve to increase secretion of adrenaline and angiotensin, which could induce vascular spasm, narrow of vascular lumen to increase insertion friction and obstruction, and led to insertion difficulty even failture(16). Previous study has shown the potential of modified patient posture in PICC catheter placement for aged patients(17).

In this study, we evaluated modified PICC catheter placement body posture for patients by comparing incidence of catheter heterotopia and complications with the traditional posture and the results suggest that there is no statistical difference in both incidences of catheter heterotopia and complications in control and experiment group which further confirms the feasibility of the modified posture for catheter placement.

\section{Effects of blocking jugular vein on catheter tip reaching expected position}

PICC catheter placement, which can be easily operated and causes fewer complications, is widely used in China. However, catheter heterotopia can still happen for many reasons. If catheter tip is not located in lower $1 / 3$ of superior vena cava, it can easily cause complications, 
such as catheter obstruction, phlebitis, infection and other problems (18). In this study, there were 25 patients with catheter heterotopia in control group. For the patients whose catheters dislocate to right atrium, we can directly adjust the catheter to proper position. However, for patients with catheters dislocating to Jugular vein, axillary vein or twist, we need to reinsert cathether.Confirmed by X-ray radiograph observation, 21 of the 25 patients were reinserted successfully. The other 4 patients whose catheter couldn't be reinserted to proper position after many attempts were revealed to have vascular abnormity. There were 17 patients with catheter heterotopia in the experiment group and they were all successfully reinserted with the assistance of B-ultrasound observation.

\section{Precautions for catheter heterotopia}

Many reasons accounted for catheter heterotopia, such as oversize straight vein, forced insertion and choice of insertion vein $(19,20)$. This study suggested comfort and relaxation of patients was important for successful insertion. And there was no relationship between insertion posture of patients and insertion success. According to anatomical feature, when catheter gets to subclavian vein, there is a branch with up to jugular vein and down to innominate vein and finally superior vena cava. Lili et al suggested that inserting PICC through cephalic vein can easily cause 
catheter to reflex because of sharp angle between cephalic vein and innominate vein (20). Among the above 42 cases of catheter heterotopia, 28 chose cephalic vein, 5 chose basilica vein and 9 chose median cubital vein. The more catheter heterotopia incidence through cephalic vein coincides with previous study. Also among the above 42 patients who had catheter heterotopia, 19 have bad vascular elasticity, 4 have vascular abnormality, 6 suffered from lung cancer and had mediastinumal oppression. Two of the 6 lung cancer patientsalso had clavicle deformation.

These observations highly suggested that in order to prevent catheter heterotopia, we need to choose proper vein based on individual anatomical features before inserting PICC. For patients with lung disease, we need to check chest radiograph first and then choose proper inserting site. In addition, operator must have good skills and follow the operation guidelines. During the operation, the patients can choose comfortable and relaxed posture for catheter insertion. The insertion should be slow to avoid catheter reflex. Meanwhile, observation through ultrasound imaging of blood vessel is a good way to reduce insertion troubles, and it can also lower complications $(21,22)$. American Center for Disease Control and Prevention also advocates that inserting PICC guided by venous ultrasound can improve insertion success and prevent (23). 


\section{CONCLUSION}

Modified patients' posture during PICC catheter placement procedure can not only save operation time and simplify procedure, but also make patients feel more comfortable and relaxed to increase their obedience. Especially for patients with dementia, Parkinson disease, cerebral infarction and other mental disorders, modified position should be more widely used in clinical practice.

\section{ACKNOWLEDGMENTS}

Thanks very much for all the patients participated in the study.

\section{Conflict of interest statement}

No conflict of interest is declared by the authors. 


\section{REFERENCES}

1. Xing H, Jia Y. Peripherally inserted central catheter (PICC) routine in clinical care. (in Chinese) Shanghai: Shanghai Jiaotong University Press 2005;2-4.

2. Tilton D. How to fine-tune your PICC care. RN 2006;69: 30.

3. Zhao H, Wang P. PICC in neurosurgical patients and nursing care (in Chinese) Qilu Nursing 2006;12:731.

4. Weijun S, Yuying L.108 patients outside the hospital management of patients with PICC chemotherapy. (in Chinese) Qilu Nursing 2006;12:53.

5. Deng X, Feng Y. PICC catheter in patients of ICU experience. (in Chinese) Guangxi Medical 2006;8:62.

6. Chunhui Z, Wang and Qiang C. Peripherally inserted central catheter puncture complications and prevention. (in Chinese) Journal of Nursing 2004;39:700-2.

7. Hsiang-Jung C, Xiaoya W, Li L. The peripherally inserted central venous catheter in 14 cases of ectopic Analysis and Prevention. (in Chinese) Liberation Army Nursing Journal 2005;

22:68-9.

8. Tamburro RF, Conner DT, Proctor KE, Bulter CL, Britton LR. The effect of a mediastinal mass on the initial positioning of a peripherally inserted central venous 
catheter. J Infus Nurs 2003;26:92-6.

9. Racadio JM, Doellman DA, Johnson ND, Bean JA, Jacobs B. Pediatric peripherally inserted central catheters: complication rates related to catheter tip location. Pediatrics 2001;107: E28.

10. Mitsufuji N, Matsuo K, Kakita S, Ikuta H. Extravascular collection of fluid around the vertebra resulting from malpositioning of a peripherally inserted central venouscatheter in extremely low birth weight infants. J Perinat Med 2002;30: 341-4.

11. Ya Z, Xuedi G. under ECG monitoring of peripherally inserted central venous catheter cardiac catheter to reduce the incidence of the effect of ectopic. Liberation Army Nursing 2010;27:1711-2.

12. Gamulka B, Mendoza C and Connolly B. Evaluation of a unique, nurseinserted, peripherally inserted central catheter program. Pediatrics 2005;115:1602-1606.

13. Ohki Y, Tabata M, Kuwashima M, Takeuchi H, Nako Y, Morikawa A. Ultrasonographic detection of very thin percutaneous central venous catheter in neonates. Acta Pediatr 2000;89:1381-4.

14. Bilong F, Shuyuan Y, Sujun Z. ECG change during PICC procedure and its application(in Chinese) China nursing magazine 2010; 45:26-8. 
15. Ying L, Hujun E, Jiang Ze. The digitized stomach and intestines X-ray machine in correction of the PICC dystopia and the localization applied research. (in Chinese) nursing journal 2010;17:59-61.

16. Juan W, Ya-hong C. Comparison between peripheral vein central venous catheter and the femoral vein catheter. (in Chinese) Journal of Nursing 2000;16: 40.

17. Keqin W. PICC puncture in elderly patients improved method. (in Chinese) Journal of Modern Nursing 2005;14:1314.

18. Michelle E, Thanh H, Nguyen. Optimizing the patient positioning for PICC linetip determination. Emergency Rediology 2005;10:186-9.

19.Hsiang-Jung C, Xiaoya W, Ni L. Peripherally inserted centralvenous catheter caused complications and its nursing care (in Chinese). Journal of Nursing 2003;18:844-5.

20. Li L,Min R, Jin-I C. PICC catheter care of complications during the intervention. (in Chinese) Journal of Practical Traditional Chinese Medicine 2004;17:389.

21. Xiu-hua Z, ZHAO, Shu-so Z. B Ultrasound-guided PICC line observation and analysis of the effect of puncture. (in Chinese) Critical Care Medicine 2006;9:1748.

22. Feng-ping S, Hongmei L, Yan C. B Ultrasound-guided PICC catheternursing. (in Chinese) Liberation Army Nursing Journal 2008;25:64. 
23. June H,Lan G, Tang Xiang Y. Two-dimensional color Doppler flow imaging in the application of PICC catheters and its effect. (in Chinese) Journal of Nursing 2007;42:739-41. 
Table 1: Patients distribution of control and experiment group

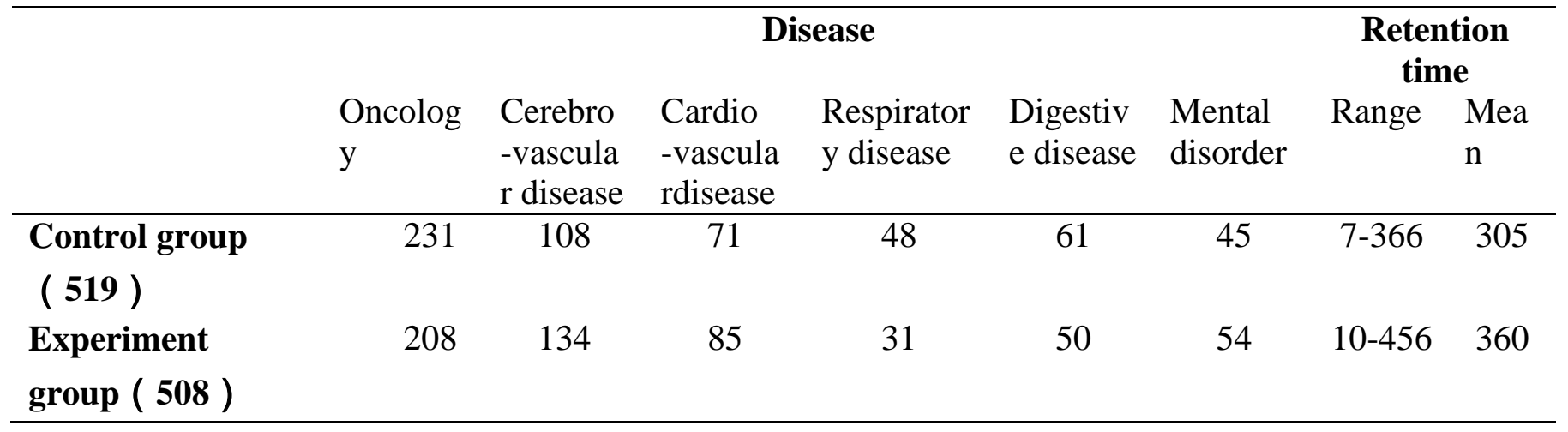

Table 2: General information of patients between control and experiment group

\begin{tabular}{|c|c|c|c|c|c|c|}
\hline & Items & $\begin{array}{l}\text { Sample } \\
\text { size }\end{array}$ & $\begin{array}{c}\text { Control group } \\
\qquad(\mathrm{n}=519)\end{array}$ & $\begin{array}{c}\text { Experiment } \\
\text { group } \\
(\mathbf{n}=508)\end{array}$ & $\chi^{2}$ & $p$ \\
\hline \multirow{2}{*}{ Age } & Less than 50 years & 434 & $223(51.38 \%)$ & $211(48.62 \%)$ & \multirow{2}{*}{0.216} & 0.64 \\
\hline & 50 or more & 593 & $296(49.92 \%)$ & $297(50.08 \%)$ & & 2 \\
\hline \multirow{2}{*}{ Gender } & Male & 622 & $301(48.39 \%)$ & $321(51.61 \%)$ & \multirow{2}{*}{2.898} & Q مि \\
\hline & female & 405 & $218(53.82 \%)$ & $187(46.18 \%)$ & & 9 \\
\hline \multirow{2}{*}{ Inserted arm } & Left & 622 & $301(48.39 \%)$ & $321(51.61 \%)$ & \multirow{2}{*}{2.892} & 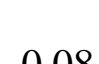 \\
\hline & Right & 405 & $218(53.82 \%)$ & $187(46.18 \%)$ & & 9 \\
\hline \multirow{4}{*}{ Inserted vein } & basilic vein & 787 & $398(50.57 \%)$ & $389(49.43 \%)$ & 0.084 & $\begin{array}{c}0.08 \\
4\end{array}$ \\
\hline & median cubital vein & 212 & $101(50 \%)$ & $101(50 \%)$ & \multirow{5}{*}{1.134} & \\
\hline & cephalic vein & 27 & $13(48.15 \%)$ & $14(51.85 \%)$ & & \\
\hline & Jugular vein & 11 & $7(63.64 \%)$ & $4(36.36 \%)$ & & \multirow{3}{*}{$\begin{array}{c}0.48 \\
8\end{array}$} \\
\hline \multirow{2}{*}{$\begin{array}{c}\text { Treatment } \\
\text { protocol }\end{array}$} & Chemo-treatment & 398 & $200(50.25 \%)$ & $198(49.75 \%)$ & & \\
\hline & parenteral nutrition & 376 & $198(47.34 \%)$ & $178(52.66 \%)$ & & \\
\hline
\end{tabular}


Table 3: Complications of patients between control group and experiment group

\begin{tabular}{|c|c|c|c|c|c|c|c|c|}
\hline & & Cathete & & Cathe & & Inserti & & \\
\hline $\begin{array}{c}\text { Sam } \\
\text { ple }\end{array}$ & Phle & r & $\begin{array}{l}\text { Skin } \\
\text { aller }\end{array}$ & ter & Infec & on tot & incide & $\chi^{2}$ \\
\hline size & & tion & gy & ion & & m & & \\
\hline
\end{tabular}

$\begin{array}{llllllllll}\begin{array}{c}\text { Control } \\ \text { group }\end{array} & 519 & 7 & 11 & 3 & 2 & 2 & 15 & 40 & 7.71 \%\end{array}$

Experim

ent

$508 \quad 7$

8

0

1

$\begin{array}{lllcc}8 & 25 & 4.92 \% & 2.86 & 0.80 \\ 4 & 0\end{array}$

group

Tota

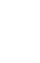

Total $1027 \quad 14$

19

3

3

323

$23 \quad 65 \quad 6.33 \%$

Table 4: Incidences and positions of catheter heterotopia between two groups

\begin{tabular}{|c|c|c|c|c|c|c|c|c|}
\hline & \multirow{2}{*}{$\begin{array}{l}\text { Sampl } \\
\text { e size }\end{array}$} & \multicolumn{3}{|c|}{ Heterotopia } & \multicolumn{4}{|c|}{ Positions of heterotopia } \\
\hline & & yes & no & incidence & $\begin{array}{c}\text { Jugular } \\
\text { vein }\end{array}$ & $\begin{array}{c}\text { Axillary } \\
\text { vein }\end{array}$ & $\begin{array}{c}\text { Right } \\
\text { atrium }\end{array}$ & Twist \\
\hline $\begin{array}{l}\text { Control } \\
\text { group }\end{array}$ & 519 & 25 & 494 & $4.8 \%$ & 9 & 3 & 7 & 6 \\
\hline $\begin{array}{c}\text { Experiment } \\
\text { group }\end{array}$ & 508 & 17 & 491 & $3.0 \%$ & 4 & 4 & 5 & 4 \\
\hline$\chi^{2}$ & & & 2.383 & & & & & \\
\hline$p$ & & & 0.123 & & & & & \\
\hline
\end{tabular}

